\title{
Diseño didáctico para propiciar el aprendizaje
}

\author{
Didactic design to cause the learning
}

Margarita Rosa Rendón Femández

Universidad de la Salle. Colombia. mrendon@unisalle.edu.co

\section{Resumen}

En el marco de la investigación formativa surge este proyecto, del cual, solo se presenta el avance de la segunda fase. El objetivo es proponer un diseño didáctico que propicie el aprendizaje de la química por medio de estrategias didácticas con base en las intencionalidades formativas del área. Implica el diseño e implementación de estrategias de enseñanza y aprendizaje que promuevan el desarrollo de saberes, habilidades, destrezas y actitudes desde las tres dimensiones (contextual, procesual y cognitiva) que conforman el diseño didáctico propuesto. De esta forma, se espera que la implementación y evaluación del diseño didáctico en el espacio académico de química, permitan completar el proceso y hacer la revisión correspondiente para verificar la validez de la propuesta diseñada.

\section{Palabras clave}

Diseño didáctico, estrategias pedagógicas, metacogniaión y aprendizaje.

\section{Abstract}

Within the framework from the formative investigation east project arises, from which, the advance of the second phase only appears. The objective is to propose a didactic design that causes the learning of chemistry by means of didactic strategies with base in the formative intentionalities of the area. It implies the design and implementation of education strategies and leaming that promote the development of saberes, abilities, skills and attitudes from the three dimensions (contextual, procesual and mental) that conform the proposed didactic design. Of this form, one hopes that the implementation and evaluation of the didactic design in the academic space of chemistry, allow to complete the process and to make the revision corresponding to verify the validity of the designed proposal.

\section{Key words}

Didactic design, pedagogical strategies, metacognición and learning.

\section{Introducción}

La necesidad de proponer un diseño didáctico para indagar acerca de las diferentes teońas que se han ocupado de la forma en que los seres humanos aprenden no sólo desde la revisión de los conocimientos que aprehenden sino de cómo estos se incorporan y desarrollan en el marco de procesos formativos. En esta reflexión es válido que se analice desde el ejercicio docente no sólo como profesionales que enseñan sino como seres que aprenden; recordar la forma en se adquiere los conocimientos que se posee y cómo el paso de la vida permite un buen ejercicio para entender que todos los seres humanos reproducen esquemas de enseñanza y aprendizaje adquiridos a través de modelos de quienes nos formaron. 


\section{Justificación}

El aprendizaje de la Química es un tema de gran preocupación para los ingenieros y para los docentes que pretenden ofrecer un espacio de verdadera construcción puesto que los estudiantes de ingeniería no ven la importancia de aplicar su ingenio en nuevos procesos.

Por tanto el curso de Química basado en la propuesta curricular del Departamento de Ciencias Básicas desde la pregunta ¿Cuál es el sentido de la formación básica, dirigida a los estudiantes universitarios, dentro del marco de un pensamiento análogo al científico?, propende por mejorar la problemática por medio de la implementación del modelo didáctico.

Por otra parte el Objeto de estudio de la química visto como los modelos de construcción y los modelos de la estructura de la materia para comprender los fenómenos naturales y los provocados por el hombre

Por lo anterior la construcción del syllabus tiene muchos elementos que propenden por que los estudiantes conozcan las normas de seguridad y se apropien del manejo y prevención de riesgos en los laboratorios y del majo de materiales y residuos, además que puedan expresar su conocimiento de manera escrita por medio de reportes cientúficos en donde se encuentre análisis y condusiones de su proceso en el laboratorio, además del trabajo en equipo en donde se pueda observar su creatividad.

\section{Descripción de la población}

El modelo se implemento en tres aursos de primer semestre Grupo 09, $011 y 013$ en el espacio académico llamado Química que tienen estudiantes de las cinco facultades de ingeniería de la Universidad de La Salle (ingeniería Ambiental, Alimentos, Diseño, Civil y Eléctrica). Cada curso conto con 20 estudiantes en promedio y sus edades estaban entre los 16 y los 18 años.

El propósito del curso es mostrar la Química, como la ciencia que está en el centro de muchos aspectos que preocupan a la sociedad, como son la preservación del medio ambiente, la producción de alimentos, la fabricación de nuevos materiales electrónicos, el descubrimiento de vacunas, la fabricación de medicamentos, etc.

Es así como el conocimiento y aplicación de los principios químicos, permite al profesional de las diferentes ingenierías, llegar a comprender como está constituida la materia, como se clasifica, que propiedades la caracterizan y como aprovechar este conocimiento para transformarla.

Es fundamental por tanto que las personas encargadas de tomar decisiones posean conocimientos cient́ficos en alguna medida. Un curso de química para estudiantes que no se van a especializar en este campo, deben dar énfasis en las aplicaciones prácticas de la química, a problemas relacionados con la contaminación ambiental, la alimentación, la radioactividad, las fuentes de energía, la salud humana y la escasez de materiales.

\section{Diseño metodológico}

La investigación acción educativa es un tipo de investigación cualitativa ayyo objetivo central es proporcionar elementos para escoger la mejor respuesta a determinadas situaciones que acontecen en el ambiente escolar. En este sentido, es una reflexión sobre la acción docente, un método para tender puentes entre teoría y práctica que se caracteriza por reflexionar sobre el hecho educativo en el corto, mediano o largo plazo con el propósito de intervenir para mejorar. La 
intervención exige que los actores educativos involucrados en una situación problema profundicen en el diagnóstico de tal manera que enriquezcan la comprensión del problema y en consecuencia elijan la mejor acción a seguir.

La Investigación Acción Educativa está diseñada para trabajar sobre problemas que se presentan en la vida cotidiana y que demandan pronta solución; antes que un tipo de investigación interesado en el "saber" por el "saber" o en fenómenos de estudio poco articulados con la realidad del día a día, la IAE funciona y tiene sentido de ser en contextos donde se requieren soluciones a partir de un conocimiento que parta de los actores involucrados en los contextos.

En otras palabras, la realidad determina la objetividad de los estudios educativos. Esto quiere decir que los diferentes actores son quienes conocen mejor los problemas que afectan directamente la calidad de la educación. Esta premisa es importante, especialmente, porque la educación se manifiesta en acciones, las cuales viven los maestros diariamente y es sobre ella que debe investigarse.

Desde estos postulados se han diseñado los fundamentos teóricos (Modelo didáctico para la formación en la autonomía) y los instrumentos (Diseño didáctico - syllabus, ficha de información personal, ficha de evaluación final del curso, diario de campo del docente, guías de aprendizaje, fichas de evaluación y notas promedio obtenidas por los estudiantes) que servirán como medios para obtener y sistematizar la información de la apuesta didáctica realizada.

\section{UNIVERSIDAD DE LA SALLE}

SYLABUS

PROGRAMA:

Espacio Académico (asignatura):

Profesor responsable:

Número de estudiantes:

Horario:

Fundamentos curriculares

(dimensión contextual)

Este espacio debe ser diligenciado teniendo en cuenta que el estudiante conozca la temáticas propias del curso en su interrelación con las demás que conforman su plan de estudios, las intencionalidades de formación del curso, el perfil que se espera tener de él al finalizar sus estudios, el devenir histórico de la disciplina que estudia y las relaciones de esta con su contexto vital.

Descle la perspectiva del docente, esto implica justificar la integración de su asignatura con el plan de estudios del programa y el perfil de formación del estudiante.

Preguntas que hacen intencionada la formación

Se refiere a la formulación de preguntas que orientan y articulan el proceso formativo.

Competencias privilegiadas por el programa

Las competencias explicitadas en este acápite serán las propias de cada institución

educativa de acuerdo con sus sentidos e intereses.

Principios, valores y actitudes del PEUL (proyecto educativo institucional) que aborda

el espacio académico.

Deben ser formulados de acuerdo con los valores, principio educativos y propósitos de

formación que se ha propuesto la institución y serán fortalecidos desde el desarrollo

de cada curso.

Diseño didáctico

(planeador de metodología de aprendiraje por contenido)

\begin{tabular}{l|l|l} 
Contenido & Objetivos/logros & Estrategias de
\end{tabular} de aprendizaje enseñanza

Estrategias de aprendizaje
Estrategias de Evaluación

Bibliografía:

Observaciones: 
Como técnica de recolección de la información se ha tomado la observación participante; la cual busca interpretar un grupo de acontecimientos y los comportamientos que se producen dentro del mismo con el fin de establecer cómo interfieren estos en las realidad de las comunidades, en nuestro caso como docente titulare de la asignatura de química, y el papel se centró en ser orientador del proceso y observador de cómo se fue desarrollando este en el marco del modelo didáctico.

Inicialmente, se desarrolló toda la fundamentación teórica del modelo para la formación en la autonomía, posteriormente se elaboró un instrumento para la realización del diseño didáctico de la asignatura de química, el cual se expresó dentro del Syllabus asumido por el departamento, para la planeación del curso. Este formato (syllabus) permitió articular los presupuestos institucionales, las intencionalidades formativas y la planeación general del curso.

\section{Métodos y técnicas para la implementación y evaluación del modelo didáctico para la formación en la autonomía}

Como una forma de organizar la secuenciación e integración de los elementos propuestos en el modelo hemos diseñado un instrumento que además de permitir la planeación de la asignatura, integre y articule: temáticas/contenidos del aurso, logros, propósitos u objetivos de formación, estrategias de enseñanza, estrategias de aprendizaje y estrategias de evaluación.

\section{DISEÑO DIDÁCTICO PARA LA FORMACIÓN EN LA AUTONOMÍA}

Contenido

Objetivos

logros de

aprendizaje

Estrategias

de

ensefianza

Estrategias

de

aprendizaje

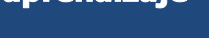

Hace referencia a los contenidos que se van a trabajar en la sesión. Es necesario tener claro a qué tipo de conocimiento se refiere: conceptual, procedimental, actitudinal, y que habilidades 0 actitudes se van a potenciar con el fin de establecer las estrategias de enseñanza y aprendizaje más adecuadas a los propósito de formación. Un objetivo es la manifestación de un propósito, una finalidad, y está dirigido a alcanzar un resultado, una meta, o objetivos de aprendizaje es necesario tener en cuenta el saber previo de los estudiantes en relación con el tema, el tipo de conocimiento que se va a aprender, así como las habilidades de aprendizaje que se pretenden potenciar.

Se refieren a los planes de acción que el docente diseña, con el fin de facilitar los procesos de aprendizaje por parte de los estudiantes. Deben estar articuladas con los objetivos de aprendizaje y los logros que se esperan alcanzar.

Existen diversidad de alternativas didácticas que facilitan el desarrollo de estos procesos, sin embargo, su utilidad dependerá de su adecuada selección, aplicación y retroalimentación.

Son actividades, ejercicios o procedimientos mediante los cuales se espera propiciar en el estudiante un proceso de reflexión y metacognición que le permitan hacerse conciente de los elementos y procesos involucrados en el desarrollo de sus aprendizajes. Por otra parte, facilitan al estudiante la identificación de su estilo de aprendizaje y del tipo de técnicas o ejercicios que más facilitan su comprensión y aprehensión de los saberes, habilidades y actitudes.

Algunas estrategias:

- Para explorar saberes previos (Preguntas, formulación de propósitos, indagación de motivos, lluvia de ideas, matriz CQA.

- Para organizar conocimientos (mapa conceptual, cuadro sinópticos, mentefactos, clasificadores, tablas de contenidos)

- Para el desarrollo de habilidades comunicativas (lectura autorregulada, resumen, escritura autorregulada, exposición)

- Para el desarrollo de habilidades interpersonales (aprendizaje en pequeños grupos, aprendizaje cooperativo, técnica del rompecabezas) entre muchas otras. 
Debe ser diseñada de acuerdo con los objetivos de aprendizaje, el proceso de Aprendizaje realizado y la verificación de los saberes y habilidades de aprendizaje

Estrategias

de desarrolladas.

La estrategia de enseñanza seleccionada sirve como mecanismo de evaluación del

Evaluación proceso y puede ser complementada con algún tipo de preguntas o ejercicios que permitan evidenciar el proceso realizado por el estudiante y el desarrollo de las habilidade Algunos instrumentos de evaluación:

- Rúbrica de evaluación del desempeño (matrices que con base en los objetivos de aprendizaje y los procesos realizados buscan verificar lo aprendido por el estudiante y el proceso que éste ha realizado con el fin de mejorar, ajustar, mantener)

- Evaluación tipo test; prácticas, laboratorios, entre otras.

\section{Análisis de la información}

Los instrumentos (Diseño didáctico - syllabus, ficha de información personal, ficha de evaluación final del curso, diario de campo del docente, guías de aprendizaje, fichas de evaluación y notas promedio obtenidas por los estudiantes) permitieron, reunir información de carácter cuantitativo y cualitativo, por ejemplo las percepciones de los estudiantes frente al desarrollo de las guías y su proceso de aprendizaje, también los resultados procesuales desde la perspectiva de los profesores.

Para la sistematización de la información se realizará un proceso de cruce de la información desde dos fuentes. La primera se constituye en los datos encontrados en la ficha personal y ficha de evaluación final, en las cuales se recogen datos relacionados con las percepciones de los estudiantes antes y después del proceso, así como información relacionada con sus conocimientos y expectativas frente al desarrollo de la asignatura. Esta información permite mostrar, por otra parte, la utilidad de la aplicación del diseño mediante las guías de aprendizaje desde la perspectiva de los estudiantes. La segunda fuente de información se centra en datos procesuales desde la percepción de los docentes, éstas surgen de la reflexión permanente basada en la aplicación de las guías y el cumplimiento de los objetivos de formación propuestos.

\section{Resultados}

El proceso comenzó con la implementación de una prueba diagnóstica a 41 estudiantes que arrojo los siguientes datos:

A nivel de los aspectos académicos se encontró que los estudiantes manifestaron que las temáticas a tratar en el curso son la tabla periódica, el estudio de la química, el estudio de la materia y por último que se desarrollará un complemento de lo visto en el bachillerato pero se percibe que un gran número de ellos no conocen los contenidos del aurso.

En cuanto a las expectativas del curso dedararon que les permitirá complementar lo que no traen del bachillerato, desarrollar aptitudes que les ayuden en el proceso de construcción de la carrera, adarar dudas y profundizar en los temas y por último obtener bases para los siguientes semestres.

Como resultado a la pregunta del significado del curso para su formación, dedararon la importancia del laboratorio con respecto al manejo y comportamiento de las sustancias con las que van a trabajar, la posibilidad de dar explicación de los fenómenos ambientales, energéticos de alimentos y muchos más. Es la base para otras asignaturas que en el futuro requieren retomar los fundamentos vistos y para aplicar lo visto en alguna problemática que se presente en el futuro. 
En lo que concieme a los procesos de evaluación que se les facilita la mayoría de los estudiantes expresaron estar de acuerdo con los exámenes con libro abierto y con trabajos en grupo, en cuanto a las evaluaciones preguntas de falso y verdadero, las de preguntas abiertas o las de preguntas de memoria no les interesa para nada

Finalmente en lo que tiene que ver con los momentos de auto, $\infty 0$ y heteroevaluación un $90 \%$ de los estudiantes afirman que favorecen los procesos de aprendizaje, porque permite una retroalimentación permanente entre el estudiante los compañeros y el docente, además escribieron que, permite conocer el proceso, lo que se está mejorando, las falencias y los desempeños a nivel individual y grupal.

Para lograr el propósito del curso se construyeron 6 guías de aprendizaje las cuales presentan una metodología de tres momentos que se van a mantener en todas las guías: Una actividad básica en la cual el trabajo es a nivel individual para que el estudiante pueda evidenciar los conocimientos que tiene en cada tema o las ideas previas, una actividad practica que consiste en trabajo en equipo que requiere la capacidad de escuchar, explicar, adarar, contraponer y defender su propuesta y finalmente proponerla en la plenaria, un último memento denominado actividad de aplicación en donde se requiere un trabajo por parejas donde explican la solución a las situaciones o ejercicios propuestos. Las guías se van relacionando unas con otras, puesto que para poder avanzar se requiere retomar conceptos vistos en las guías anteriores.

\section{Conclusiones}

El hecho de incluir las guías de aprendizaje como instrumento metodológico para la implementación del diseño didáctico propuesto permitió evidenciar:

A pesar de que todos los docentes desarrollan un proceso de planificación inicial para la gestión de sus asignaturas, este se asume más como un requisito que como una guía permanente que oriente las acciones pedagógicas aplicadas en el aula, en este sentido, se evidencia, a través del desarrollo de este proyecto, que las guías se convierten en una mediación fundamental para la planeación, sistematización y evaluación del proceso pedagógico, resultando funcional tanto para docentes como para estudiantes.

$>$ El ejercicio de escritura en la elaboración de las guías permite al docente reflexionar en tomo al proceso, lograr una mayor apropiación del conocimiento lo cual facilita, establecer una dinámica que promueve el dialogo entre docentes y estudiantes y contribuye a la cualificación permanente del proceso.

La aplicación del modelo a través del diseño didáctico propuesto se fundamenta en el ejercicio consciente y permanente de habilidades de aprendizaje que a pesar de su naturaleza compleja se ponen de manifiesto en el desarrollo de acciones configuradas a partir de diferentes estrategias diseñadas en función de los objetivos de formación.

Por otra parte, como experiencia de investigación acción en el aula, la formulación, aplicación y evaluación del modelo didáctico, permitió profundizar en enfoques teóricos y metodológicos que permitieron adarar y apostarle a una interrelación conciente y sistematizada de los enfoques curriculares, los modelos pedagógicos y los modelos didácticos desde una mirada integradora y contextualizada, lo que equivale a decir desde un proceso sistemático, riguroso y con muchas posibilidades de ser probado, mejorado y utilizado como mecanismo eficaz para cualificar el proceso educativo. 


\section{Bibliografía}

Amnestoy, M. (1998), Desarrollo de habilidades de pensamiento. Procesos directivos, ejecurtivos y de adquisioión de conocimiento, México, Trillas

Ausubel, D, Novak, J y Hanesian, H. (1983), Educational psychology. A cognitive view, new cork, holt trad. México, Trillas.

Barrios, P. (1992), Propuesta de un programa de entrenamiento a docentes en estrategias cognoscitivas para la comprensión de lectura con niños de educación primaria. [Tesis de licenciatura] México, UNAM Facultad de Psicología.

Bell, 0. (1971). Thinking about the Curriaulum. Escuela general Noruega. Oslo.pp.91

Bellmont, J.M (1989), "Cognitive strategies and strategic learning" en: American psychologist, 44 (2). pp. 142-148.

Beltrán, J. (1987). Aprender a aprender: desarrollo de estrategias cognitivas. Madrid, Cincel.

Bruner, J. (1988). Desarrollo cognitivo y educación, Madrid, Morata.

Buron Orejas, Javier. (1998). Enseñar a aprender. Introducción a la metacognición Bilbao, Mensajero.

Cárdenas et al. (2002) El concepto de competencia I Una mirada interdisciplinar: Bogotá. Edit. Magisterio.

Dansereau, D.F. (1985), Leaming strategy research, en: J.W. Segal, S.E. Chipman y R Glaser (Eds) Thinking and learning skills. Hillsdales, N.J. Lawrence Eribaum.

De Bono, E. (1983), "the cognitive research tust (cort) thinking program", en w. Maxwell (comp.), thinking: the expanding frontier. Philadelphia. The franklin institute press.

De la Torre, Saturnino. et al. (2002), Estrategias didácticas innovadoras, Barcelona, Octaedro.

Díaz B. y Hemández, G. (1998) Estrategias docentes para un aprendizaje significativo. México, Mograw Hill.

Diaz Barriga, F, Hemández Rojas, G. (1999), Estrategias docentes para un aprendizaje significativo. México, Mograw Hill. 